\title{
Aplicación de un modelo sistémico para el diseño conceptual de un asistente abdominointestinal.
}

\author{
Esnal-Angulo, Iñaki ${ }^{a}$ \& Hernandis-Ortuño, Bernabéb. \\ a $\mathrm{PhD}$ candidate, Universitat Politècnica de València, Spain. iaesan@doctor.upv.es. \\ ${ }^{\mathrm{b}}$ PhD Full Professor, Universitat Politècnica de València, Spain. bhernand@upv.es.
}

\begin{abstract}
Resumen
El artículo describe una investigación mediante la aplicación de un modelo sistémico orientado al diseño conceptual de un producto, que actúa como asistente abdominointestinal, permitiendo reducir el estreñimiento crónico funcional en las personas, debido a la adopción de la postura de cuclillas en su uso, así como a la realización de ejercicios específicos de tonificación. La metodología de la investigación consistió en la construcción de un escenario de simulación estática mediante la implementación de un modelo de formulación por objetivos (Hernandis, 2003) en el que los parámetros y variables del sistema actúan como atributos y/o variables de diseño. El proceso de identificación de dichas variables se llevó a cabo mediante investigación exploratoria y el análisis descriptivo y cualitativo basado en las diversas fuentes documentales. Los trabajos de S. Raad (1994) sobre la variación del ángulo del músculo puborrectal (encargado de la continencia) en diferentes configuraciones posturales del paciente y el ensayo de usabilidad de I. Esnal y C. Serrano (2013) sobre el nivel de satisfacción del usuario en función de la postura adoptada, sirvieron para establecer algunos de los objetivos más relevantes de la investigación. Tras la identificación y definición de las variables, éstas fueron clasificadas en tres subsistemas fundamentales: subsistema funcional, subsistema ergonómico y subsistema formal. Posteriormente, se generaron los volúmenes de uso, las superficies de uso y los límites de contorno de estas variables mediante su geometrización. Con la superposición de estas geometrías se creó un espacio volumétrico de diseño en el que se representa el sistema en estudio, aportando tangibilidad en forma de concepto de producto. Como resultado de la investigación se obtuvo un marco teórico y el espacio geométrico de diseño único donde crear un concepto de producto capaz de satisfacer los objetivos impuestos por el sistema y por lo tanto, cumplir con los requerimientos de diseño.
\end{abstract}

Palabras clave: Modelo sistémico, diseño, dispositivo, asistente, estreñimiento. 


\begin{abstract}
This paper describes a research by the application of a systemic model oriented to the design of a product. This device works as abdominointestinal assistant allowing reduce chronic functional constipation in people due to the adoption of the squatting posture and specific toning exercises in its use. Research methodology consisted of the construction of a static simulation scenario by implementing a goal-directed model (Hernandis, 2003) in which system parameters and variables act as attributes/design variables. The identification of these variables was carried out by using exploratory research and qualitative analysisbased on of the various documentary sources. The works of S. Raad (1994) on the variation of the angle of puborectal muscle (responsible for continence) in different postural configurations of the patients and the usability test of I. Esnal and C. Serrano (2013) on the level of user satisfaction depending on the posture adopted, served to establish the main aims of the research. After identification and definition, the variables were classified into three main subsystems: functional subsystem, ergonomic subsystem and formal subsystem. Subsequently, volumes of use, surfaces of use and boundaries were established by their geometries. By the superposition of these geometries it was created a space of design in which we can represent the system under study. This provides tangibility to the system in the form of product concept. Another result we found is the theoretical framework of design. It provides an unique three-dimensional geometric space of design in which we can generate product concepts satisfying the aims set by the system and therefore, the design requirements.
\end{abstract}

Keywords: Systemic model, product design, constipation, squatting.

\title{
1. Introducción
}

El estreñimiento (o constipación) es un problema que afecta a millones de personas en todo el mundo especialmente en países occidentales. De acuerdo con la Organización Mundial de la Salud (OMS, 2010) el 12\% de la población mundial padece esta afección y en España, según la Asociación Española de Gastroenterología (AEG, 2013) el número asciende a más de 6 millones de personas.

Sólo en EEUU, en función de la definición empleada ${ }^{74}$ se da entre el $2 \%$ y el $27 \%$ de la población (Lembo, A. \& Camilleri, M., 2003), lo que conlleva más de 2,5 millones de visitas al especialista y 92 mil hospitalizaciones. Los costes totales que esto acarrea se estiman en 6,9 mil millones de dólares anuales, de los cuales 725 millones se destinan a los productos laxantes. Por otro lado, en Reino Unido en 2006 los médicos generales emitieron más de 13 millones de recetas para fármacos destinados a paliar esta afección. (OMGE, 2010).

Pese a todo esto, el estreñimiento habitualmente puede ser manejado a nivel de atención primaria con control costo-efectivo de los síntomas. Si bien esta afección no amenaza la vida ni debilita al individuo y en la mayoría de casos sólo produce molestia, en algunas ocasiones puede acarrear consecuencias de

\footnotetext{
${ }^{74}$ Criterios Roma III. (Zolezzi, A., 2007)
} 
mayor gravedad derivando en otras patologías o afecciones como: la diverticulosis, la colitis, la apendicitis, el prolapso rectal o el cáncer de colon.

Una variante de éste, el estreñimiento crónico funcional, es un trastorno mecánico que posee dos fisiopatologías diferenciadas: los trastornos del tránsito que pueden ser causado por una disminución del movimiento intestinal (de tránsito lento) o por una disfunción del suelo pélvico (Van Endelenburg, 2013); o por trastornos de la evacuación, causado por la pérdida de la sensibilidad de los músculos rectales y/o del esfínter (Amir, A., 2013).

\section{Estado del arte}

Para llevar a cabo la investigación se han contemplado dos campos diferentes, por un lado, el relacionado con la afección mediante la profundización en el estudio, en particular, del estreñimiento funcional inducido fundamentalmente por la atrofia del suelo pélvico por ser ésta una de las causas directas de mayor influencia en este tipo de trastorno disfuncional del colon. Y por otro lado se ha considerado el estudio de los modelos sistémicos, más concretamente los orientados al diseño, en especial, al diseño de producto; como herramienta y método para la consecución del desarrollo de una propuesta conceptual de solución o paliativo de la problemática propuesta.

\subsection{Estreñimiento funcional y la postura de cuclillas}

Existen autores que proponen paliar esta afección de la manera más natural posible como es la adopción de la postura de cuclillas. Su visión es que la vida sedentaria y pasiva, en general, y la irrupción del inodoro de postura sedente en la sociedad moderna como arraigo cultural en particular, está afectando a muchas personas empeorando los síntomas o desarrollándolos.

B. Wallace en 2002, describe una serie de comportamientos que suceden a nivel interno a consecuencia de la adopción de la postura de cuclillas y por los cuales el autor considera que dicha postura favorece la evacuación: "El peso del torso presiona los muslos y de forma natural comprime la parte superior del intestino [...], cierra la válvula ileocecal y presuriza el colon facilitando con ello el desplazamiento de la materia fecal a través del mismo [...]. El músculo puborrectal, normalmente encargado de preservar la continencia, se relaja y permite la elevación del colon sigmoide aumentando el ángulo del pliegue y ensanchando la entrada del recto". La magnitud de este reflejo fue cuantificado por S. Rad (2002) quien publicó un estudio en el cual, mediante la radiografía, midió los ángulos de los músculos de los pacientes durante la defecación. En dicho ensayo se les pidió a los pacientes que usaran ambos tipos de inodoro, el occidental (postura sedente) y el tradicional-oriental (postura de cuclillas) y poder así medir la diferencia existente entre los ángulos de los músculos que intervienen y si esto afectaba o no a la eficiencia en la evacuación. El ensayo le permitió concluir que el músculo puborrectal se relaja y aumenta el ángulo en un $40^{\circ}$ de media entre un caso y otro.

Por otro lado, I. Esnal y C. Serrano en 2013 publican un estudio biomecánico comparativo (Esnal, I. \& Serrano, C. 2013) entre ambas posturas durante la evacuación. El estudio fue llevado a cabo mediante un ensayo de usabilidad con el uso de un prototipo estático diseñado para simular ambas opciones posturales. A pesar de no poseer un número de población muestral significantemente elevado (45 usuarios, 21 hombres y 24 mujeres), concluyeron que existía cierta tendencia a la mejoría con la postura de cuclillas en determinadas alturas, asumiendo así la existencia de cierta relación entre la postura adoptaba y la eficiencia (volumen) y el grado de satisfacción (sensación de vacío) por parte del usuario. De este estudio, se extrajeron las condiciones iniciales relacionadas con la postura de cuclillas, como la posición (ángulos 
y longitudes) de los segmentos corporales de los miembros inferiores del usuario, implícitas en mayor medida en variables como la proporción y la usabilidad.

\subsection{Pensamiento sistémico orientado al diseño de productos.}

La gestión del conocimiento bajo el enfoque más amplio de la sistémica induce a considerar nuevas formas de interrelación de la información, resultando de ello nuevas posibilidades de configuración entre los elementos y, contribuyendo así, al aumento del grado de innovación en los resultados obtenidos. Este aumento de la información gestionada aporta un nuevo marco teórico de mayor complejidad, pero a su vez y con las herramientas adecuadas, de mayor grado de control, lo cual permite poseer mayor precisión en el proceso de modelización de los sistemas.

B. Hernandis (2000) publica un modelo sistémico orientado al diseño de productos industriales basado en los esquemas de control por objetivos jerarquizados de Ashby-Melèse y el trabajo de L. Ferrer, Presidente de Honor Permanente de la Sociedad Española de Sistemas Generales (SESGE). Dicho modelo propone la simbiosis entre el estudio de los sistemas y el diseño industrial, de modo en el que el producto es considerado un sistema en sí mismo. La estructuración interna que rige este modelo se representa mediante tres subsistemas fundamentales equivalentes en jerarquía o isosistemas, en el que cada uno contempla un tipo de información dependiendo de la naturaleza de ésta. El subsistema funcional recoge toda la información referida a los aspectos tecnológicos y estructurales del producto, como: la resistencia, los materiales, los procesos de fabricación, etc.. El subsistema ergonómico contempla todos los aspectos derivados de la relación entre el objeto y el usuario: la adaptabilidad, usabilidad, confort, psicología, etc... Y el subsistema formal encargado de considerar los aspectos relacionados con la visualización: proporción, morfología, color, textura, estética, estilo, etc...

El uso de esta perspectiva para el estudio de sistemas orientados al diseño, y más concretamente al de producto, se encuentra cada vez en más ámbitos de esta disciplina. Prueba de ello, encontramos autores centrados en los aspectos metodológicos como son J. Briede \& Hernandis (2011) quienes estudiaron mediante la sistémica las primeras fases del proceso creativo y conceptual, orientado al diseño de producto y a la enseñanza (Briede \& Hernandis, 2009). A A. Medina et al. (2012) en la extracción de los aspectos diferenciales del producto, fundamentalmente centrado en el producto joya o M. Cabello (2009), con el estudio de los factores diferenciales del diseño gráfico en el etiquetado en el sector vinícola. Estos estudios, si bien no están orientados a los productos sanitarios, sirvieron como referencia para establecer tanto los aspectos fundamentales a considerar como el grado de detalle durante el proceso de modelización. B. Hernandis y J. Bonmati (2005) mediante el uso del análisis estructural de un estudio de opinión, cuantificaron la importancia de estos aspectos fundamentales en la definición del producto. Establecieron el grado de motricidad y de dependencia de las variables como indicadores para conocer las relaciones entre ellas y su grado de participación. De este estudio se extrajo un listado de diferentes autores sobre los factores determinantes a la hora de establecer los requerimientos de diseño y que ha servido de referencia en esta investigación (Tabla 1). 
Tabla1. Factores determinantes para establecer los requerimientos de diseño

\begin{tabular}{|c|c|c|c|}
\hline $\begin{array}{l}\text { G. Bonsiepe / G. } \\
\text { Rodríguez }\end{array}$ & $\begin{array}{c}\text { Gómez-Senent y } \\
\text { Capuz }\end{array}$ & B. Munari & S. Pugh \\
\hline 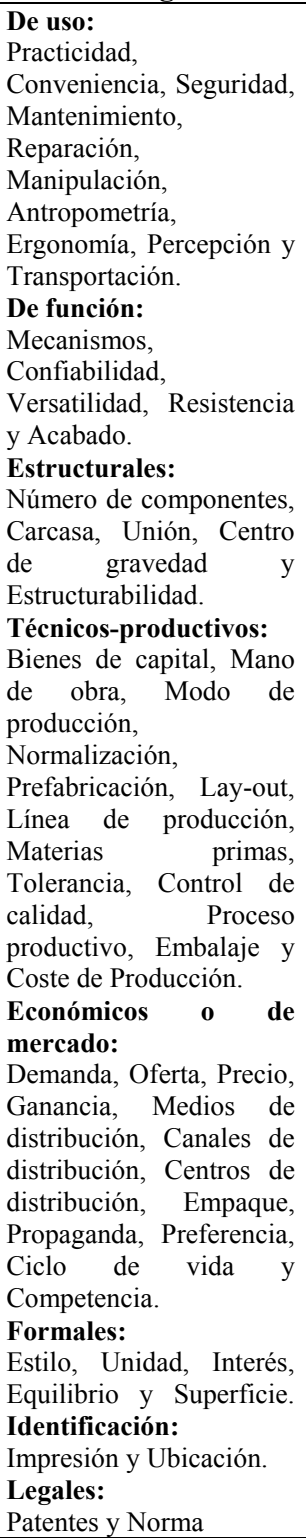 & $\begin{array}{l}\text { Fundamentales: } \\
\text { Tecnológico, Materiales, } \\
\text { Económico, Seguridad, } \\
\text { Ergonómico, Estético, } \\
\text { Mantenimiento } \\
\text { De entorno: } \\
\text { Seguridad } \\
\text { Normas } \\
\text { Medio Ambiente Cliente } \\
\text { Competitividad } \\
\text { Equipamiento } \\
\text { Instalaciones Volumen de } \\
\text { producción Rendimiento } \\
\text { de la planta } \\
\text { Proceso de producción } \\
\text { Patentes } \\
\text { Riesgo del proyecto } \\
\text { Envasado Expedición } \\
\text { Capacidad de los } \\
\text { proveedores } \\
\text { Materiales } \\
\text { Coste del producto } \\
\text { Peso y tamaño } \\
\text { Documentación } \\
\text { Mantenibilidad } \\
\text { Valor y utilidad } \\
\text { Estética } \\
\text { Calidad Ergonomía } \\
\text { Erado }\end{array}$ & $\begin{array}{l}\text { Nombre del objeto } \\
\text { Autor } \\
\text { Productor } \\
\text { Dimensiones } \\
\text { Materia } \\
\text { Peso } \\
\text { Técnicas } \\
\text { Coste } \\
\text { Embalaje } \\
\text { Utilidad declarada } \\
\text { Funcionalidad } \\
\text { Ruido } \\
\text { Mantenimiento } \\
\text { Ergonomía } \\
\text { Acabados } \\
\text { Manejabilidad } \\
\text { Duración } \\
\text { Toxicidad } \\
\text { Estética } \\
\text { Moda (Styling) Valor } \\
\text { social } \\
\text { Esencialidad } \\
\text { Precedentes } \\
\text { Aceptación por parte del } \\
\text { público. }\end{array}$ & $\begin{array}{l}\text { Performance } \\
\text { Environment } \\
\text { Life in service } \\
\text { (performances) } \\
\text { Maintenance } \\
\text { Target product cost } \\
\text { Competition } \\
\text { Shipping } \\
\text { Packing } \\
\text { Quantity appearance } \\
\text { Manufacturing facility } \\
\text { Size life } \\
\text { Weight span } \\
\text { Aesthetics, and } \\
\text { and finish } \\
\text { Materials } \\
\text { Product literature and } \\
\text { Standards } \\
\text { specifications } \\
\text { Ergonomics Customer } \\
\text { Quality and reliability } \\
\text { Shelf life (storage) } \\
\text { Processes } \\
\text { Time-scales } \\
\text { Testing Safety } \\
\text { Company constraints } \\
\text { Market } \\
\text { Patents, lonstraints } \\
\text { product data Political and } \\
\text { Social implications } \\
\text { Legal Installation } \\
\text { Documentation Disposal }\end{array}$ \\
\hline
\end{tabular}

Fuente: Hernandis, B. \& Bonmatí, J. (2005)

S. Paixao et al, (2012) dirige su enfoque al estudio de los materiales, más concretamente a la piedra, proponiendo nuevos usos de los materiales naturales. Junto con E. Merino en 2009 también estudiaron la aplicación de la sistémica en el diseño concurrente, especialmente con la ergonomía como parte integrante del sistema.

J. Rivera, J. R. González \& B. Hernandis. (2013) publican un estudio del contexto y las variables conceptuales para la sostenibilidad del producto dentro del modelo sistémico. Cardozo et al. (2013) por otra parte, se centra en la personalización, la variabilidad y la diferenciación de los sistemas de producto. Los mismos autores en 2015 propusieron la categorización de los sistemas de productos según el uso y la experiencia del consumidor. 
Mediante el uso del Análisis de Componentes Principales realizado sobre un estudio cuantitativo de opinión a usuarios y de valoración de expertos, B. Agudo et al., (2016), confirman una vez más, la existencia de tres componentes principales en los que se pueden reagrupar los atributos de diseño: Función, Ergonomía y Forma. Además, ofrece una herramienta de garantía para el estudio de la trazabilidad en los sistemas de producto.

Teniendo en cuenta los diferentes enfoques desde los que se puede abordar el estudio de los sistemas orientados al diseño y todos los aspectos considerados por las fuentes estudiadas, especialmente las centradas en el producto, se propuso el uso de los modelos sistémicos como una metodología propicia para la consecución del cumplimiento de los requerimientos de diseño necesitados para abarcar una problemática tan compleja.

\section{Metodología}

La investigación realizada consistió en la construcción de un escenario de simulación estática mediante la implementación de un modelo sistémico de formulación por objetivos orientado a productos (Hernandis, 2003) (Fig. 1) en el que los parámetros y variables del sistema son interpretados como atributos y/o variables de diseño; y en donde los subsistemas no están jerarquizados, sino que son isosistemas de igual importancia.

Este escenario contempla el conjunto "Universo de posibles" (U) integrado por dos sistemas fundamentales o suprasistemas: el Sistema Exterior (SE), que actúa como entorno; y el Sistema en estudio (SES), referido al sistema de referencia, en este caso un producto de asistencia abdominointestinal.

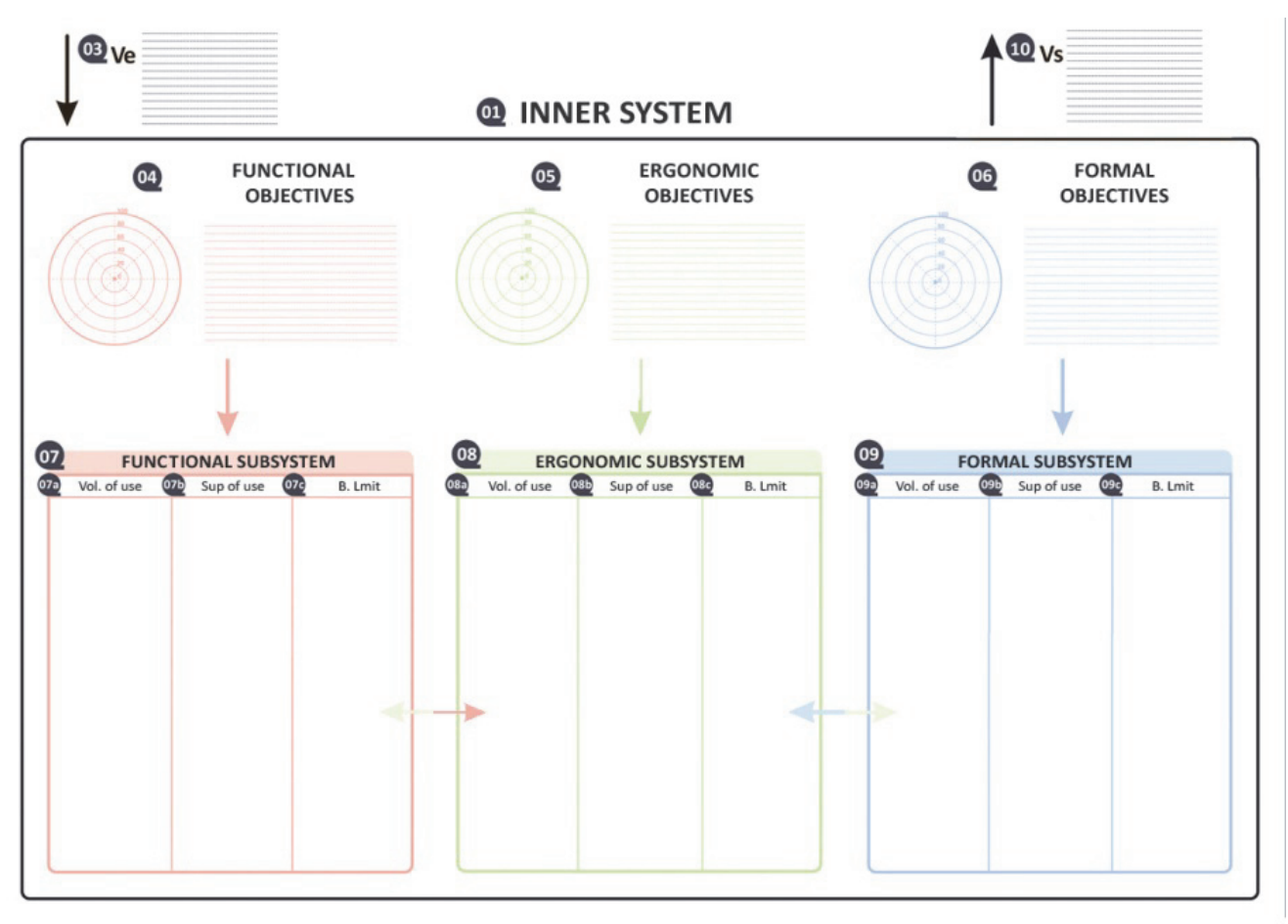

Fig. 1 Modelo sistémico de producto por objetivos. Adaptado de Hernandis (2003) 
Mediante investigación exploratoria por literatura de las diversas fuentes documentales, se identificaron las variables exógenas que conforman el conjunto Sistema Exterior o entorno. Para ello se hizo especial hincapié en aquellos aspectos que poseen una mayor participación y, por lo tanto mayor grado de influencia, en el diseño y desarrollo de productos según las fuentes consultadas.

El análisis descriptivo y cualitativo de dicha información permitió la extracción, síntesis y clasificación de la misma, representando con ello la información que viaja desde el Sistema Exterior al interior del Sistema en Estudio y constituyendo así las variables de entrada necesarias para modelar dicho Sistema en Estudio. En la figura 2 se muestra la estructura interna del modelo, las trayectorias y el flujo de la información desde el Sistema Exterior o entorno al Sistema en Estudio y su comportamiento dentro de éste.

Para la identificación y constitución de las variables nos ayudamos, como se menciona en el apartado anterior, de diversas fuentes, como por ejemplo para la definición de la variable de entrada correspondiente a la proporción, derivada en parte, de la configuración postural que ha de adoptar el usuario (postura de cuclillas), fue extraída de los resultados aportados por el ensayo de usabilidad realizado por I. Esnal \& C. Serrano (2013) donde estimaron una altura apropiada de elevación de los pies del usuario en función de los ángulos adoptados por los diferentes segmentos corporales inferiores. Esta estimación se reduce a un rango de entre $10-22 \mathrm{cms}$ de los pies con el suelo en postura sedente. Para ello, se tuvo en consideración la variación del ángulo del pliegue del músculo puborrectal (encargado de la continencia), interpretado de los estudios observacionales del radiólogo S. Raad (1994) quien determinó una apertura media de 35-40 grados y en casos excepcionales de 80 grados al adoptar la postura de cuclillas. Tras definir las variables de entrada, se establecieron, clasificaron y listaron los objetivos del sistema en los tres subsistemas fundamentales: Objetivos Funcionales, Objetivos Ergonómicos y Objetivos Formales.

Posteriormente, se realizó la geometrización de las variables que constituyen el sistema mediante la asignación de los volúmenes de uso, superficies de uso y límites de contorno en cada uno de los subsistemas fundamentales, definiendo de este modo el rango de acción y con ello construyendo los espacios de intervención en el sistema geométrico. Se ha de mencionar, la interpretación de los volúmenes como el primer paso hacia la materialización del sistema, pero cabe destacar que de igual importancia son los espacios negativos que restringen al mismo y poseen una naturaleza de índole inmaterial. Mediante la suma de estas geometrías creamos un espacio geométrico de diseño en el que poder representar el conjunto global de variables del Sistema en Estudio y en el cual concebir un concepto de producto aportando mayor tangibilidad al sistema.

\section{Resultados}

Los primeros resultados de la investigación fueron los listados de las variables externas extraídas de la síntesis de la información constituyente del Sistema Exterior y, posteriormente tras su procesamiento, las variables de entrada (Tabla1). 
Tabla 1 Listado de variables.

\begin{tabular}{|l|l|}
\hline \multicolumn{1}{|c|}{ V externas } & \multicolumn{1}{c|}{ V entrada } \\
\hline Vex1. Usuarios & Ve1. Segmento de usuarios \\
Vex2. Antropometría & Ve2. Características físicas usuario \\
Vex3. Estreñimiento & Ve3. Estreñimiento \\
Vex4. Patologías asociadas & Ve4. Patologías del usuario \\
Vex5. Mecanismos & Ve5. Mecanismos \\
Vex6. Materiales & Ve6. Materiales \\
Vex7. Sostenibilidad & Ve7. Reciclabilidad \\
Vex8. Procesos de fabricación & Ve8. Procesos de fabricación \\
Vex9. Usabilidad & Ve9. Facilidad de uso \\
Vex10. Mercado & Ve10. Mercado \\
Vex11. Legislación & Ve11. Psicología \\
& Ve 12. Normativa \\
\hline
\end{tabular}

Como uno de los resultados principales se obtuvo el modelo sistémico de producto único para un asistente abdominoinstestinal de ayuda a reducir el estreñimiento funcional mediante la adopción de la postura de cuclillas. En él se comprenden las variables de entrada y salida, los atributos / objetivos de los subsistemas y los propios subsistemas fundamentales (Fig. 3).

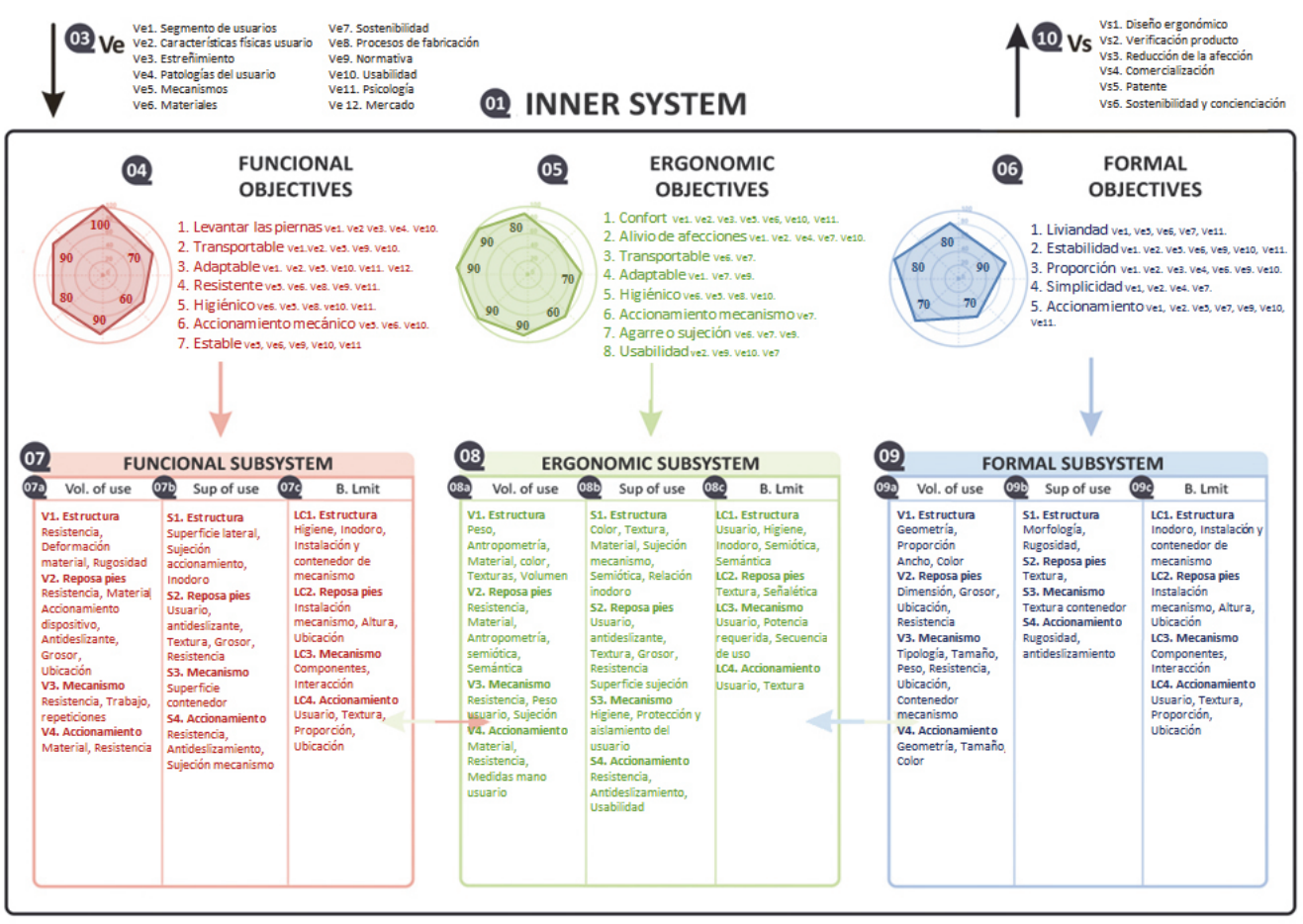

Fig. 3 Modelo sistémico para el diseño de un asistente abdominointestinal. 
Para una mejor visualización de los resultados, éstos se han dividido en diferentes tablas.

La tabla 2 corresponde al listado de los atributos obtenido tras la recolección y valoración de los datos que sirvieron como referencia a la hora de establecer los objetivos principales del Sistema en Estudio.

Tabla 2 Listado de Atributos:

\begin{tabular}{|c|c|c|}
\hline $\begin{array}{c}\text { Atributos } \\
\text { Funcionales }\end{array}$ & $\begin{array}{c}\text { Atributos } \\
\text { Ergonómicos }\end{array}$ & $\begin{array}{l}\text { Atributos } \\
\text { Formales } \\
\end{array}$ \\
\hline 1. Elevar las piernas & 1. Confort & 1. Liviandad visual \\
\hline 2. Resistente & 2. Alivio de afecciones & 2. Estabilidad visual \\
\hline 3. Adaptable & 3. Transportable & 3. Proporción \\
\hline 4. Estable & 4. Adaptable & 4. Simplicidad \\
\hline 5. Transportable & 5. Higiénico & 5. Accionamiento mecánico \\
\hline 6. Higiénico & 6. Accionamiento manual & 6. Estética mimética \\
\hline 7. Accionamiento manual & 7. Agarre o sujeción & \\
\hline 8. Sostenible & 8. Usabilidad & \\
\hline 9. Seguro & 9. Seguridad & \\
\hline 10. Desmontable & 10. Fácil manipulación & \\
\hline 11. Fácil manipulación & & \\
\hline
\end{tabular}

Mediante la asignación de volúmenes, superficies y límites de contorno, se obtuvo un listado (Tabla 3) en el que se muestran cada uno de estos elementos para cada uno de los subsistemas fundamentales. Así mismo se muestran también las variables involucradas directa o indirectamente con los espacios que las representan. 
Aplicación de un modelo sistémico para el diseño conceptual de un asistente abdominointestinal.

Tabla 3. Volúmenes de uso, Superficies de uso y Límites de contorno

\begin{tabular}{|c|c|c|c|c|c|c|c|c|}
\hline \multicolumn{3}{|c|}{ Subsistema Funcional } & \multicolumn{3}{|c|}{ Subsistema Ergonómicos } & \multicolumn{3}{|c|}{ Subsistema Formales } \\
\hline Vol. de uso & Sup. de uso & $\mathbf{L C}$ & Vol. de uso & Sup. de uso & $\mathbf{L C}$ & Vol. de uso & Sup. de uso & $\mathbf{L C}$ \\
\hline $\begin{array}{l}\text { V1. Estructura } \\
\text { Resistencia } \\
\text { Deformación } \\
\text { Material } \\
\text { Proporciones }\end{array}$ & $\begin{array}{l}\text { S1. Estructura } \\
\text { Sup. Lateral } \\
\text { Sujeción } \\
\text { Acción } \\
\text { Inodoro }\end{array}$ & \begin{tabular}{|l|} 
LC1. Estructura \\
Higiene, \\
Inodoro, \\
Instalación y \\
contenedor de \\
mecanismo
\end{tabular} & $\begin{array}{l}\text { V1. Estructura } \\
\text { Peso } \\
\text { Antropometría } \\
\text { Material } \\
\text { Color } \\
\text { Texturas, } \\
\text { Volumen, }\end{array}$ & \begin{tabular}{|l|} 
S1. Estructura \\
Color \\
Textura \\
Material \\
Sujeción \\
mecanismo \\
Semiótica \\
Relación inodoro
\end{tabular} & \begin{tabular}{|l|} 
LC1. Estructura \\
Usuario \\
Higiene \\
Inodoro \\
Semiótica \\
Semántica
\end{tabular} & $\begin{array}{l}\text { V1. Estructura } \\
\text { Geometría, } \\
\text { Proporción } \\
\text { Ancho, Color }\end{array}$ & $\begin{array}{l}\text { S1. Estructura } \\
\text { Morfología, } \\
\text { Rugosidad }\end{array}$ & $\begin{array}{l}\text { LC1. Estructura } \\
\text { Inodoro } \\
\text { Instalación y } \\
\text { contenedor de } \\
\text { mecanismo }\end{array}$ \\
\hline $\begin{array}{l}\text { V2. Reposa pies } \\
\text { Resistencia } \\
\text { Material } \\
\text { Acción } \\
\text { Antideslizante } \\
\text { Grosor, } \\
\text { Ubicación }\end{array}$ & \begin{tabular}{|l|} 
S2. Reposa pies \\
Usuario \\
Antideslizante \\
Textura \\
Grosor \\
Resistencia
\end{tabular} & \begin{tabular}{|l} 
LC2. Reposa \\
pies \\
Instalación \\
mecanismo \\
Altura \\
Ubicación
\end{tabular} & $\begin{array}{l}\text { S2. Reposa pies } \\
\text { Usuario } \\
\text { Antideslizante } \\
\text { Textura } \\
\text { Grosor } \\
\text { Resistencia } \\
\text { Sujeción }\end{array}$ & $\begin{array}{l}\text { S2. Reposa pies } \\
\text { Usuario } \\
\text { Antideslizante } \\
\text { Textura } \\
\text { Grosor } \\
\text { Resistencia } \\
\text { Superficie } \\
\text { sujeción }\end{array}$ & \begin{tabular}{|l|} 
LC2. Reposa pies \\
Textura \\
Señalética
\end{tabular} & $\begin{array}{l}\text { V2. Reposa pies } \\
\text { Dimensión, } \\
\text { Grosor, } \\
\text { Ubicación, } \\
\text { Resistencia }\end{array}$ & $\begin{array}{l}\text { S2. Reposa pies } \\
\text { Textura, } \\
\text { S3. Mecanismo } \\
\text { Textura } \\
\text { contenedor }\end{array}$ & $\begin{array}{l}\text { LC2. Reposa pies } \\
\text { Instalación } \\
\text { mecanismo } \\
\text { Altura } \\
\text { Ubicación }\end{array}$ \\
\hline $\begin{array}{l}\text { V3. Mecanismo } \\
\text { Resistencia } \\
\text { Trabajo } \\
\text { Repeticiones }\end{array}$ & $\begin{array}{l}\text { S3. Mecanismo } \\
\text { Sup. Contenedor }\end{array}$ & $\begin{array}{l}\text { LC3. Mecanismo } \\
\text { Componentes, } \\
\text { Interacción }\end{array}$ & $\begin{array}{l}\text { S3. Mecanismo } \\
\text { Higiene } \\
\text { Protección } \\
\text { Aislamiento del } \\
\text { usuario }\end{array}$ & $\begin{array}{l}\text { S3. Mecanismo } \\
\text { Higiene } \\
\text { Protección } \\
\text { Antideslizante }\end{array}$ & $\begin{array}{l}\text { LC3. Mecanismo } \\
\text { Usuario } \\
\text { Potencia requerida } \\
\text { Secuencia de uso }\end{array}$ & $\begin{array}{l}\text { V3. Mecanismo } \\
\text { Tipología, } \\
\text { Tamaño, Peso, } \\
\text { Resistencia, } \\
\text { Ubicación, } \\
\text { Contenedor } \\
\text { mecanismo }\end{array}$ & $\begin{array}{l}\text { S3. Mecanismo } \\
\text { Textura } \\
\text { contenedor }\end{array}$ & $\begin{array}{l}\text { LC3. Mecanismo } \\
\text { Componentes } \\
\text { Interacción }\end{array}$ \\
\hline $\begin{array}{l}\text { V4. Acción } \\
\text { Material } \\
\text { Resistencia }\end{array}$ & $\begin{array}{l}\text { S4. Acción } \\
\text { Resistencia } \\
\text { Antideslizante } \\
\text { Sujeción } \\
\text { mecanismo }\end{array}$ & $\begin{array}{l}\text { LC4. Acción } \\
\text { Usuario } \\
\text { Textura } \\
\text { Proporción } \\
\text { Ubicación }\end{array}$ & \begin{tabular}{|l|} 
S4. Acción \\
Resistencia, \\
Antideslizamiento \\
Usabilidad
\end{tabular} & $\begin{array}{l}\text { S4. Acción } \\
\text { Resistencia, } \\
\text { Antideslizante } \\
\text { Usabilidad }\end{array}$ & $\begin{array}{l}\text { LC4. Acción } \\
\text { Usuario } \\
\text { Textura }\end{array}$ & $\begin{array}{l}\text { V4. Acción } \\
\text { Geometría } \\
\text { Tamaño } \\
\text { Color }\end{array}$ & \begin{tabular}{|l|} 
S4. Acción \\
Rugosidad \\
Antideslizante
\end{tabular} & $\begin{array}{l}\text { LC4. Acción } \\
\text { Usuario } \\
\text { Textura } \\
\text { Proporción } \\
\text { Ubicación }\end{array}$ \\
\hline
\end{tabular}

De la representación geométrica, y su ensamblaje por componentes, de cada elemento resultado del listado anterior, se obtuvieron los modelos conceptuales geométricos para cada subsistema fundamental (Fig. 4). Se pueden identificar los volúmenes prismáticos generados para cada uno de ellos según los diferentes aspectos. Considerando dichos aspectos las características varían, como se puede observar en la figura, de un modelo funcional puramente recto (Fig 4, a), a un modelo más suavizado, consecuencia de una mayor adecuación o adaptación ergonómica, hasta el modelo formal, el cual muestra formas más orgánicas y de mayor expresión estética.

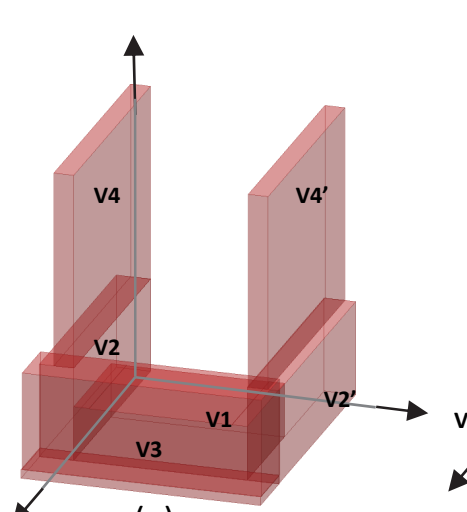

(a)

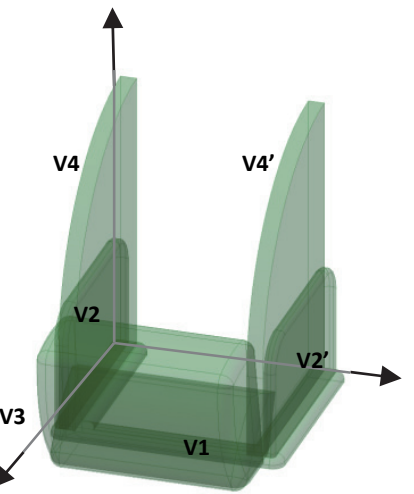

(b)

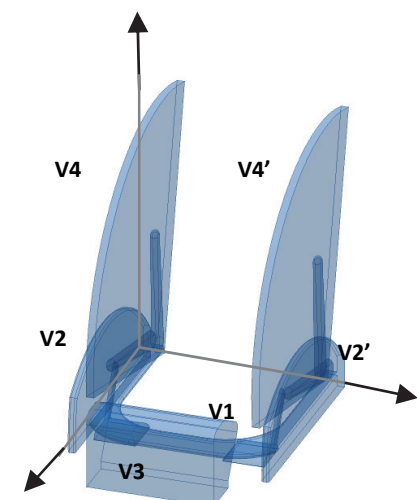

(c)

Fig. 4 Modelos conceptuales geométricos de los Subsistemas: (a) Funcional, (b) Ergonómico y (c) Formal. 
La superposición de estos modelos conceptuales geométricos da como resultado un espacio de diseño conceptual (Fig. 5) que alberga toda la información de los modelos individuales anteriormente citados y de lo que se interpreta que está sometido a las mismas consideraciones y restricciones iniciales establecidas. Por lo que se puede decir que este espacio posee las características necesarias para generar en él una propuesta de diseño que cumpla con los objetivos.

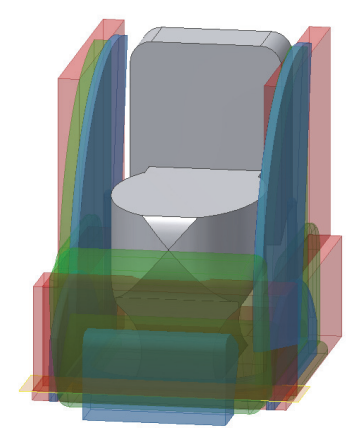

Fig. 5 Espacio de diseño para un asistente abdominointestinal.

En el espacio de diseño obtenido se generaron diferentes propuestas de diseño conceptual de las cuales se muestra una de ellas como muestra representativa (Fig. 6) para ejemplificar una de las posibles "materializaciones" del sistema.

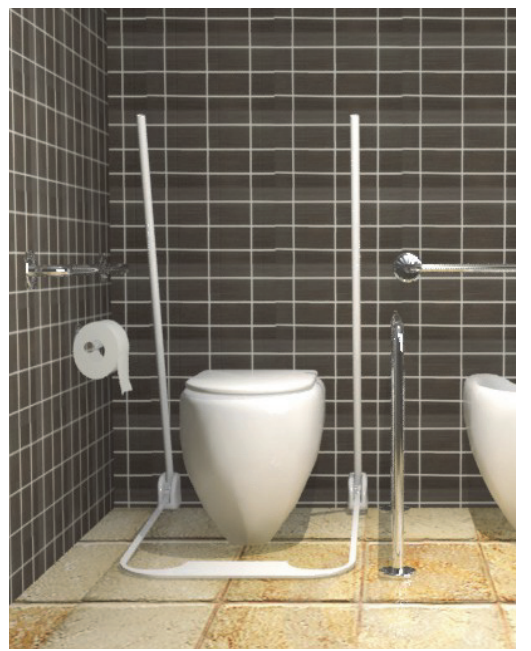

Figs. 6 Propuesta de diseño conceptual.

Para finalizar el ciclo, el Sistema en Estudio debe devolver la información adquirida de nuevo al entorno (principio homeostático), pero ésta volverá al medio procesada por el modelo como función de transformación resultando el concepto de un producto de asistencia abdominointestinal, el espacio de diseño, el marco teórico que lo alberga y las variables que lo constituyen. Esta información de salida se representa mediante las variables de salida y son el resultado de aplicar la solución de diseño al problema planteado (Hernandis, B. 2000). 
Tabla 4. Listado de las variables de salida

\begin{tabular}{|l|}
\hline \multicolumn{1}{|c|}{ V salida } \\
\hline Vs1. Diseño ergonómico. \\
Vs2. Verificación del sistema producto. \\
Vs3. Validación del modelo \\
- Vs4. Reducción de la afección. \\
- Vs5. Patente \\
- Vs6. Sostenibilidad y concienciación \\
\hline
\end{tabular}

Estas variables, enumeradas en la tabla 4, forman parte de la información referida anteriormente que sale al entorno y como sistema que es, lo modifica; éste se reconfigura y vuelve a iniciarse el ciclo de nuevo propiciando el reajuste de la información de las variables de entrada y con ello del sistema en estudio. Estos bucles son aprovechados como retroalimentación que aporta al método la flexibilidad necesaria para que el modelo se reconfigure y adapte al medio en cada ciclo. El bucle de retroalimentación finaliza cuando se alcanza el grado de cumplimiento de los objetivos establecido por el agente decisor y se considera así que el producto posee las cualidades y atributos requeridos.

\section{Conclusiones}

La visión sistémica adoptada para el estudio de la problemática como un sistema complejo permitió un abordaje de mayor amplitud y profundidad de la misma. Esto conllevó, por un lado, un incremento de la información que el diseñador como agente decisor debe gestionar y además, deberá hacerlo de la manera más eficientemente posible con el fin de cumplir con los objetivos establecidos del sistema y, por lo tanto, satisfacer los requerimientos de diseño. El propio modelo dirige la información y, con ella, se establece el flujo de trabajo que ha de seguir el modelador "obligándole" a extraer, sintetizar y entender el comportamiento de dicha información. Dota, por lo tanto, de una estructuración lógica y ordenada al proceso de diseño, especialmente en etapas donde se requiere de mucha reflexión por parte del diseñador para manejar todos los condicionantes del entorno y el cual puede, pese al incremento de la información, poseer mayor rigor en las tomas de decisión. Por otro lado, el uso de esta metodología confiere al proceso de diseño y, por consiguiente, al producto, de nuevos aspectos emergentes de las interrelaciones de la información. Lo que se traduce en cualidades innovadoras y un mayor número de soluciones.

También decir, que una de las características que posee la sistémica es que permite simular la realidad con diferentes grados de precisión y generar modelos tan detallados y complejos como se desee. El inconveniente que posee este planteamiento es que el tiempo necesario de procesamiento de la información de dicho modelo podría llegar a ser tal que el propio modelo dejara de ser de utilidad (Martínez, \& Requena, 1986). Es por esto que, en aras de favorecer el proceso de toma de decisiones, debemos plantear el problema con el grado de precisión adecuada. Debemos considerar un equilibrio entre el nivel de detalle/complejidad y mayor número de soluciones, con el tiempo de modelización o de simulación. Cabe destacar en este sentido que el caso estudiado contempla un nivel de detalle propio de 
las fases conceptuales y es por ello que se han descartado aspectos como: materiales, procesos de fabricación, normativa, costes, canales de distribución o marketing.

Es de destacar también la analogía existente con los sistemas vivos como es el principio homeostático que caracteriza a este tipo de sistemas que dicta que para mantener el estado de equilibrio, el sistema debe permanecer en constante intercambio de energía e información con el entorno en el que existe, de tal modo se sirva de los cambios en éste para retroalimentarse y reorganizarse internamente en favor de una mejor adaptación. Esto quiere decir que el propio modelo es susceptible de cualquier variación en las condiciones del entorno y por lo tanto se puede interpretar que lo mismo sucede con el producto. Este se adapta a las nuevas condiciones como lo hacen los organismos vivos en su medio.

\section{Futuras líneas de investigación}

Se pretende profundizar en el estudio del sistema "asistente abdominoinstestinal" con el fin de aumentar el nivel de detalle de las variables y parámetros de diseño. En primer lugar la investigación se centrará en las áreas correspondientes a la usabilidad del producto y su validación ergonómica. Para ello nos valdremos de un prototipo del modelo técnicamente de mayor definición para su estudio con usuarios, el cual se encuentra actualmente en proceso de desarrollo.

Se realizará un estudio cuantitativo de la opinión de los posibles usuarios mediante cuestionarios y entrevistas a expertos en diseño de producto para precisar en mayor medida las cualidades y atributos más importantes y mejor valorados.

Se pretende la implementación de sistemas neuronales como medio de control en la gestión y validación de las variables; y la aplicación de herramientas de búsqueda heurística de sistemas evolutivos para la generación y optimización de las soluciones.

\section{Referencias}

AMIR, A. (2011). "Etiological factors of constipation in the elderly, with emphasis on functional causes. Eastern Mediterranean Health Journal. Vol. 17 No. 8

LEMBO, A. \& CAMILlERI, M. (2003).“Chronic Constipation”. The New England journal of medicine. Massachusetts Medical Society. 349, pp 11360-8.

BRIEDE, J.C \& HERNANDIS, B. (2009). "An educational application for a product design and engineering system using integrated conceptual models ”. Ingeniare. Revista chilena de ingeniería, 17 (3), $432-442$.

BRIEDE, J.C \& HERNANDIS, B. (2011). "New methods in design education: The systemic methodology and the use of sketch in the conceptual design stage". US-China Education Review, 8 (1), 118-128.

CABELLO, M. (2009). "Estudio de los factores de diseño gráfico de la etiqueta de vino tinto de calidad que influyen en su elección y compra: estudio cuantitativo en valencia". Tesis doctoral, Valencia: Universidad Politécnica de Valencia.

CARDOZO, J., HERNANDIS, B. \& RAMIREZ, N. (2013). "Caracterización de los sistemas de productos en el marco de la personalización, la variabilidad y la personalización. Un estudio con expertos". Ingeniare. Revista chilena de ingeniería, vol. $22 \mathrm{~N}^{\circ}$ 2, 2014, pp. 278-291

CARDOZO, J., HERNANDIS, B. \& RAMIREZ, N. (2015). “Aproximación a una categorización de los sistemas de productos: el uso y la experiencia del consumidor como configuradores". Innovar, 25(58), pp125-142. Doi 10.15446/innovar.v25n58.52438. 
Aplicación de un modelo sistémico para el diseño conceptual de un asistente abdominointestinal.

ESNAL, I. \& SERRANO, C. (2013). "Diseño y desarrollo de un prototipo para contribuir a la disminución del estreñimiento y patologías asociadas”. Tesina Final de Máster. < http://hdl.handle.net/10251/61752>. Valencia: Universidad Politécnica de Valencia.

HERNANDIS, B. (2003). "Desarrollo de una metodología sistémica para el diseño de productos industriales. Tesis doctoral no publicada, Valencia: Universidad Politécnica de Valencia.

HERNANDIS, B. \& BONMATI, J. (2005) “El diseño coherente. La correcta definición del producto". Primer Encuentro Interinstitucional de Diseño Industrial ULA, Mérida Venezuela. ISBN: 980-11-0859-2 Vol. 2

HERNANDIS, B. \& BRIEDE, J. (2009).An Educational Application for a Product design and Engineering systems using integrated conceptual models.(pp.432-442)Arica. Ingeniare. Revista Chilena de Ingeniería.

HERNANDIS, B. \& IRIBARREN, E. (2000). Diseño de nuevos productos. Una perspectiva sistémica. Valencia: Universidad Politécnica de Valencia.

OMGE (2010) "Estreñimiento: una perspectiva mundial. Guias Mundiales de la Organización Mundial de Gastroenterología. Organización Mundial de Gastroenterología.

MARTÍNEZ, S. \& REQUENA, A. (1986) Dinámica de sistemas. T.2. Modelos. Madrid:Alianza Editorial.

PAIXAO, S., HERNANDIS, B. \& MERINO, E. (2009). The role of innovation in concurrent design model (cdm) and its consequences on the aspects of competitiveness, differentiation and sustainability. iDEMi 09- Internacional Conference on Integration of Design, Engineering and Management for Innovation Publicación:. Universidade Federal do Paraná, Brasil. ISSN 2175-3768.

PAIXAO, S.; HERNANDIS, B. \& MERINO, E. (2009). "Metodologia Sistêmica aplicada ao Design Concorrente A ergonomia como parte integrante“. ERGODESIGN - Congresso Internacional de Ergonomia e usabilidade de Interfaces Humano-Tecnologia: Produto, Informaçoes, Ambiente Construído e Transporte. Universidade Federal do Paraná, Brasil. ISSN 2175-3768.

PAIXAO, S., et al, (2012) "Design in the natural Stone transformation sector: evaluating a new concept". Ingenieria Investigación, Vol. 32, No 3 , pp $82-88$.

RAD, S. (2002). "Impact of ethnic habits on Defecographic measurements". Archiv. Iranian Med. Vol. 5, No. 2, pp $115-117$.

RIVERA, J. C., GONZÁLEZ, J. R., \& HERNANDIS, B. (2013). Analysis of contexts and conceptual variables for a sustainable approach into systemic model. Relating Systems Thinking \& Design 2013 - Emerging Contexts for Systemic Design. Oslo.

VAN ENGELENBURG, M., BOLS, E., BENNINGA, M., et al. (2013). The effect of pelvic physiotherapy on reduction of functional constipation in children: design of a multicentre randomised controlled trial. BMC Pediatrics. Doi: 10.1186/1471-2431-13-112

ZOLEZZI, A. (2007). Las Enfermedades Funcionales Gastrointestinales y Roma III. Rev. gastroenterol. Perú v.27 n.2. ISSN 1022-5129. 